\title{
Perfil hematológico dos neonatos atendidos no Hospital Universitário da Universidade Federal de Santa Catarina
}

\author{
Hematologic profile of neonates attended at the Teaching Hospital of the Federal University in \\ Santa Catarina
}

\author{
Jéssica Gonçalves ${ }^{1}$ \\ Nicéia Mara A. Souzal \\ Elizabeth M. Hermes ${ }^{2}$ \\ Cleta S. C. Jesus ${ }^{3}$ \\ Márcia Maria Jordão ${ }^{4}$
}

\begin{abstract}
O hemograma do recém-nascido oferece inúmeras informações ao neonatologista e sua interpretação pode variar de acordo com a idade gestacional e o peso de nascimento da criança. Os valores de referência para essa faixa etária são de difícil obtenção e poucos estudos foram realizados nessa área, principalmente pela dificuldade de coleta sanguínea e suas implicações para o recém-nascido. O presente estudo observa o perfil hematológico dos neonatos atendidos e compara os valores obtidos com as faixas de referência encontradas na literatura. Os resultados apontam diferenças significativas entre as faixas de referência encontradas para diversos parâmetros hematológicos, de acordo com o peso e idade gestacional dos neonatos. A pesquisa mostra a importância da utilização de diferentes valores de referência para a interpretação do hemograma do recém-nascido de acordo com esses fatores. Rev. Bras. Hematol. Hemoter. 2010;32(3):219-224.
\end{abstract}

Palavras-chave: Hemograma; recém-nascido; valores de referência.

\section{Introdução}

O hemograma do recém-nascido oferece inúmeras informações relevantes ao neonatologista. Entretanto, os valores de referência para essa faixa etária são de difícil obtenção. ${ }^{1}$ Isso pode ser justificado pelo fato de o volume sanguíneo de um recém-nascido variar de $86,3 \mathrm{~mL} / \mathrm{kg}$ no recém-nascido de termo a $89,4 \mathrm{~mL} / \mathrm{kg}$ no prematuro durante os primeiros três dias de vida, ${ }^{2}$ implicando um volume total geralmente abaixo de $300 \mathrm{~mL}$ para um recém-nascido com o peso normal, o que acaba inibindo a requisição do hemograma na criança normal, devido à espoliação sanguínea advinda da coleta. ${ }^{3}$

Contribuindo para a dificuldade de obtenção desses valores, poucos são os estudos encontrados para essa faixa etária e muitos deles ainda utilizam metodologias hoje consideradas ultrapassadas. As cifras são geralmente baseadas em estudos com pequeno número de amostras. ${ }^{1}$

Em virtude disso, a faixa de referência utilizada para essa população tão especial é estimada com base nos valores de 5 a 95 percentuais obtidos dos hemogramas de crianças com mínima patologia, entendendo que estes seriam os mais próximos dos valores normais. ${ }^{1}$ Ainda assim, os resultados encontrados pelos clínicos devem ser interpretados de acordo com a idade gestacional do neonato. Caso contrário, pode haver má interpretação dos mesmos, e o diagnóstico de condições tais como as infecções podem ser negligenciados ou atrasados. ${ }^{4}$ Outro fator que deve ser considerado na interpretação do hemograma do recém-nascido é o peso de nascimento, tendo estudos anteriormente demonstrado uma diferença significativa nos valores de leucócitos sanguíneos de crianças abaixo do peso para a idade gestacional. Nestes

Hematologia. Farmacêutica Bioquímica do HU/UFSC - Florianópolis-SC.

Controle de qualidade. Farmacêutica Bioquímica do HU/UFSC - Florianópolis-SC.

Microbiologia. Farmacêutica Bioquímica do HU/UFSC - Florianópolis-SC.

Enfermagem neonatal. Enfermeira do HU/UFSC - Florianópolis-SC.

Hospital Universitário Polydoro Ernani de São Thiago - Universidade Federal de Santa Catarina - Florianópolis-SC.

Correspondência: Jéssica Gonçalves

Avenida Desembargador Vitor Lima, 410, Apto 104, Bloco A4 - Bairro Trindade

88040-400 - Florianópolis-SC - Brasil

E-mail: jessicagoncalvesufsc@gmail.com

Doi: $10.1590 / S 1516-84842010005000077$ 
pacientes, os valores de referência de neutrófilos são diferentes quando comparados aos dos recém-nascidos de peso normal. $^{5}$

Esta pesquisa procura observar o perfil hematológico da população atendida de recém-nascidos, relacionando os resultados encontrados com idade gestacional e peso de nascimento dos mesmos, apresentando-os em forma de faixas de referência estimadas e comparando-os com os valores de referência encontrados na literatura. Os dados obtidos são dos neonatos do Hospital Universitário Ernani Polydoro São Thiago, vinculado à Universidade Federal de Santa Catarina, localizado na cidade de Florianópolis, Brasil, no período de março a setembro de 2009.

\section{Casuística e Método}

Os recém-nascidos $(\mathrm{n}=101)$ para os quais o médico solicitou a realização do hemograma tiveram as amostras de sangue coletadas com o anticoagulante EDTA-K2 e enviadas ao Setor de Hematologia do Serviço de Análises Clínicas do Hospital Universitário. Após o consentimento e a autorização dos pais, os recém-nascidos foram incluídos na pesquisa. Os hemogramas foram realizados com ajuda do contador automatizado Horiba ABX Pentra DF 120 (Horiba ABX ${ }^{\circledR}$ ), que faz uma associação dos princípios de impedância elétrica, citometria de fluxo, citoquímica e espectrofotometria para a análise hematológica. ${ }^{6}$

Os contadores hematológicos automatizados, em geral, fazem a contagem de eritrócitos, leucócitos e plaquetas utilizando o método de impedância elétrica, baseado no princípio de Coulter. ${ }^{3,7}$ A porcentagem do hematócrito é baseada em um cálculo que utiliza o número total de eritrócitos juntamente com o volume corpuscular médio para sua determinação. ${ }^{3,7} \mathrm{~A}$ dosagem de hemoglobina é realizada através de uma reação colorimétrica, onde a oxidação do ferro do grupo heme leva à formação de espécies cromogênicas, as quais são dosadas por espectofotometria. ${ }^{6}$

Após a liberação do resultado parcial pela automação, todas as amostras foram avaliadas em distensão sanguínea através de microscopia ótica por dois farmacêuticos-bioquímicos, para confirmação dos resul- tados e realização da contagem diferencial de leucócitos.

As crianças de termo (com mais de 38 semanas de idade gestacional) e prematuras (com menos de 38 semanas) foram classificadas segundo a idade gestacional em semanas, peso de nascimento (extremo baixo peso: abaixo de $1.000 \mathrm{~g}$; muito baixo peso: de $1.000 \mathrm{~g}$ a $1.500 \mathrm{~g}$; baixo peso: de $1.500 \mathrm{~g}$ a 2.500 g; peso normal: acima de $2.500 \mathrm{~g}$ ) e idade em dias para realização da análise estatística. Os parâmetros avaliados foram: eritrócitos totais, hemoglobina, hematócrito, plaquetas, leucócitos totais, diferencial de leucócitos e contagem de eritroblastos. Amostras com número de eritroblastos acima de 10 para cada 100 leucócitos tiveram o número de leucócitos totais corrigidos. A análise estatística dos dados foi realizada pelo software SPSS 17.0, e foram excluídas do trabalho crianças com diagnóstico de sepse ou resultados expúrios, restando um número final de $\mathrm{n}=97$. A pesquisa foi aprovada pelo Comitê de Ética na Pesquisa em Seres Humanos (CEPSH) da Pró-Reitoria de Extensão da Universidade Federal de Santa Catarina, instituído pela Portaria $\mathrm{n}^{\circ} 0584 / \mathrm{GR} / 99$ e registrada sob número de processo 002/09 FR-237396.

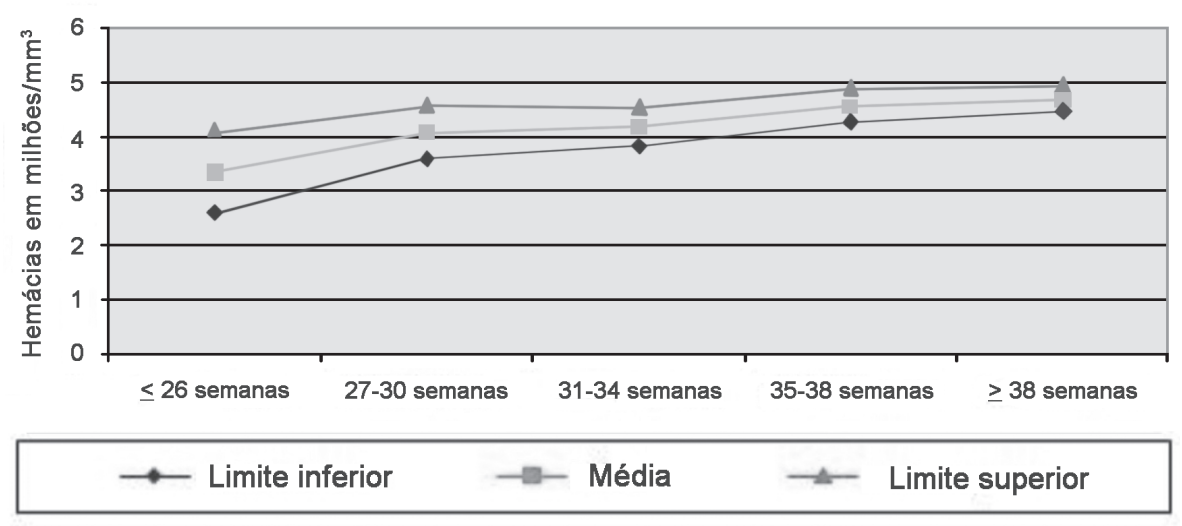

Figura 1. Faixa de referência estimada para contagem de eritrócitos em milhões $/ \mathrm{mm}^{3}$ por idade gestacional $(p<0,05):<26$ semanas: $n=8 ; 27-30$ semanas: $n=10 ; 31-34$ semanas: $n=21 ; 35$ 38 semanas: $\mathrm{n}=30$; $>38$ semanas: $\mathrm{n}=28$

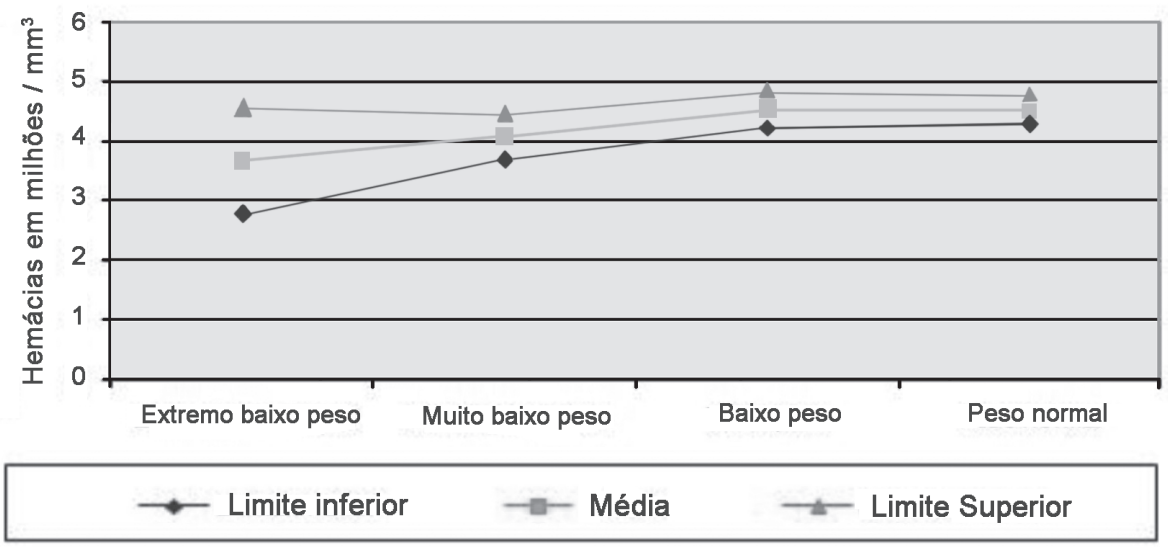

Figura 2. Faixa de referência estimada para contagem de eritrócitos em milhões $/ \mathrm{mm}^{3}$ por peso de nascimento $(p<0,05)$. Extremo baixo peso: $n=9$; muito baixo peso: $n=13$; baixo peso: $n=31$; peso normal: $n=44$ 


\section{Resultados}

Parâmetros eritrocitários

As contagens de hemácias para os recém-nascidos apresentaram diferença estatisticamente significativa $(\mathrm{p}<0,05)$ entre as faixas de idade gestacional e de peso de nascimento, e estão representadas pelas Figuras 1 e 2. Para os índices eritrocitários, as faixas de referência obtidas foram estimadas utilizando-se o intervalo de $95 \%$ de confiança, que representou melhor a população estudada. Os valores das faixas estimadas para a contagem de eritrócitos totais elevaramse de acordo com o aumento da idade gestacional e peso de nascimento dos neonatos.

$\mathrm{Na}$ análise da dosagem de hemoglobina foi observada diferença estatística $(p<0,05)$ entre as diferentes idades gestacionais; entretanto, não houve diferença para a classificação por peso. A Figura 3 apresenta as médias e limites superior e inferior para a dosagem de hemoglobina. Houve um aumento das dosagens de hemoglobina de acordo com o aumento da idade gestacional dos recém-nascidos.

A dosagem do hematócrito teve resultado semelhante à hemoglobina, apresentando diferença estatística (p $<0,05)$ somente para a classificação por idade gestacional, sendo a faixa de referência semelhante para os recémnascidos com diferentes pesos de nascimento. A Figura 4 representa a faixa de referência estimada para os valores de hematócrito, onde se observa o aumento desse parâmetro de acordo com o aumento da idade gestacional dos neonatos.

Além do anteriormente exposto, também foi feita uma análise para os índices eritrocitários classificados segundo a idade em dias dos recémnascidos (1, 2 e 3 dias de vida). Porém, não houve diferença para as faixas encontradas, ao contrário do que outro estudo havia relatado. ${ }^{8}$

$\mathrm{Na}$ análise relacionada à presença de hemácias nucleadas no sangue periférico dos neonatos, obtivemos

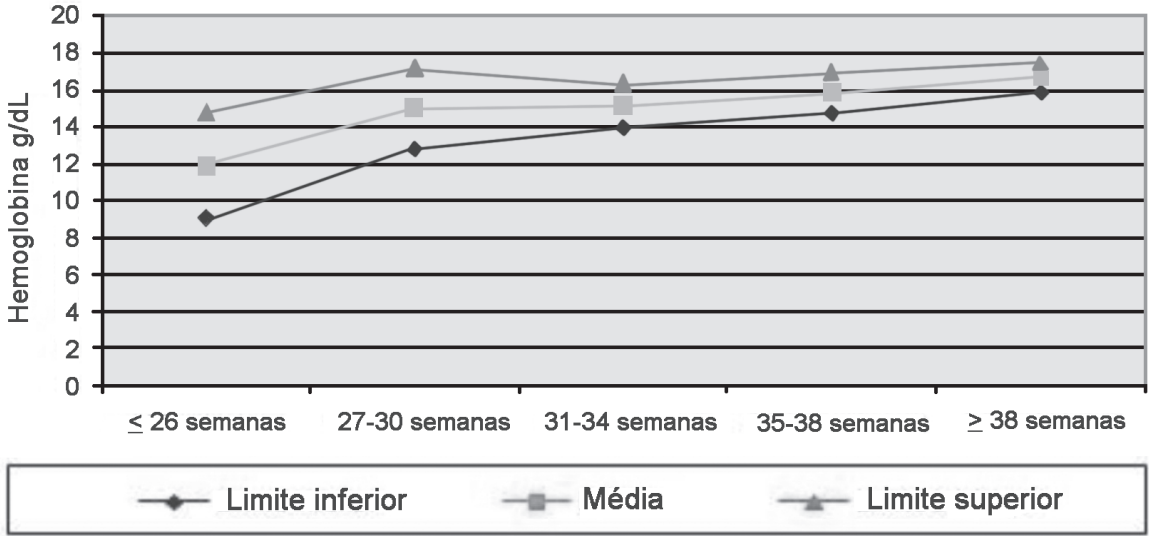

Figura 3. Faixa de referência estimada para dosagem de hemoglobina em $\mathrm{g} / \mathrm{dL}$ por idade gestacional $(p<0,05):<26$ semanas: $n=8 ; 27-30$ semanas: $n=10 ; 31-34$ semanas: $n=21$; 35-38 semanas: $\mathrm{n}=30$; $>38$ semanas: $\mathrm{n}=28$

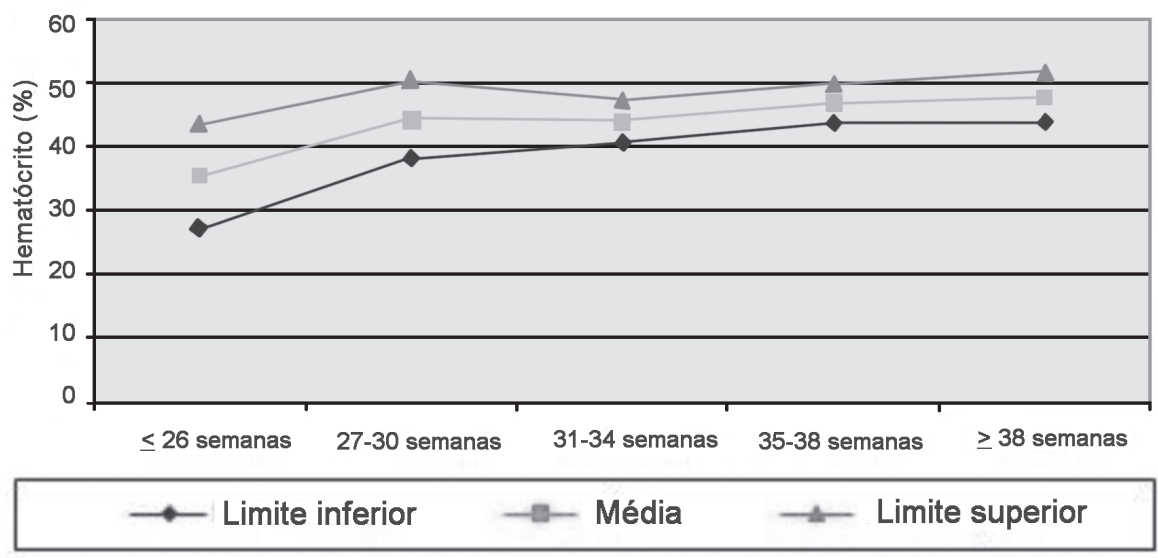

Figura 4. Faixa de referência estimada para porcentagem de hematócrito por idade gestacional $(p<0,05):<26$ semanas: $n=8 ; 27-30$ semanas: $n=10 ; 31-34$ semanas: $n=21 ; 35-38$ semanas: $\mathrm{n}=30$; $>38$ semanas: $\mathrm{n}=28$

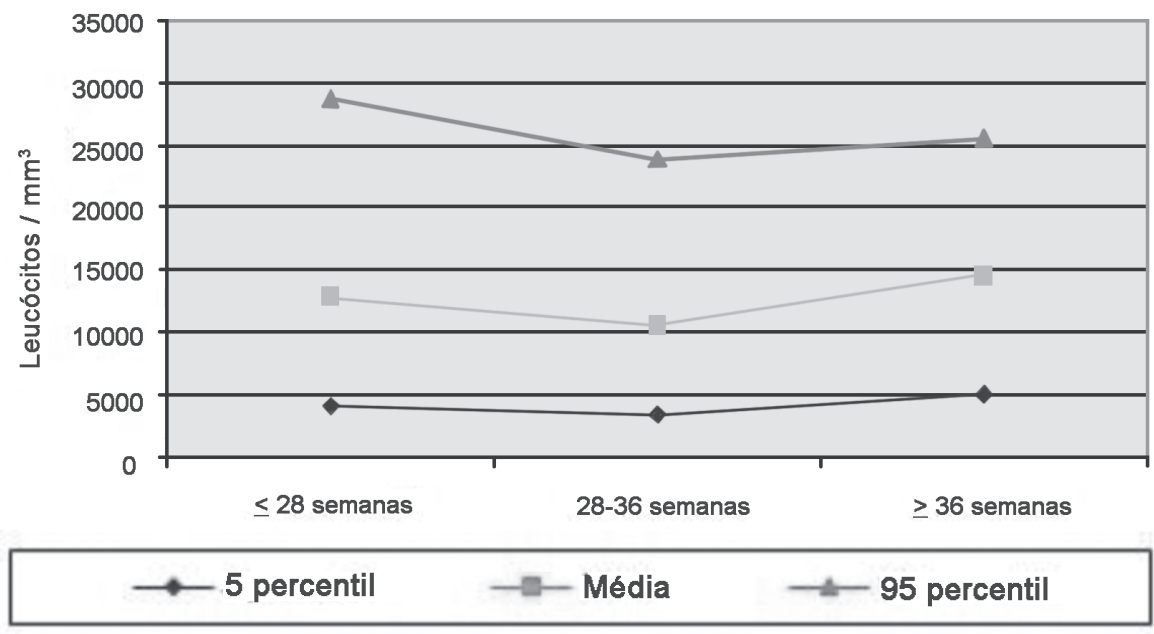

Figura 5. Faixas de referência estimadas para contagem de leucócitos totais $/ \mathrm{mm}^{3}$ por idade gestacional $(p<0,05)$ : $<28$ semanas: $n=12 ; 28-36$ semanas: $n=44 ;>36$ semanas: $n=41$ 


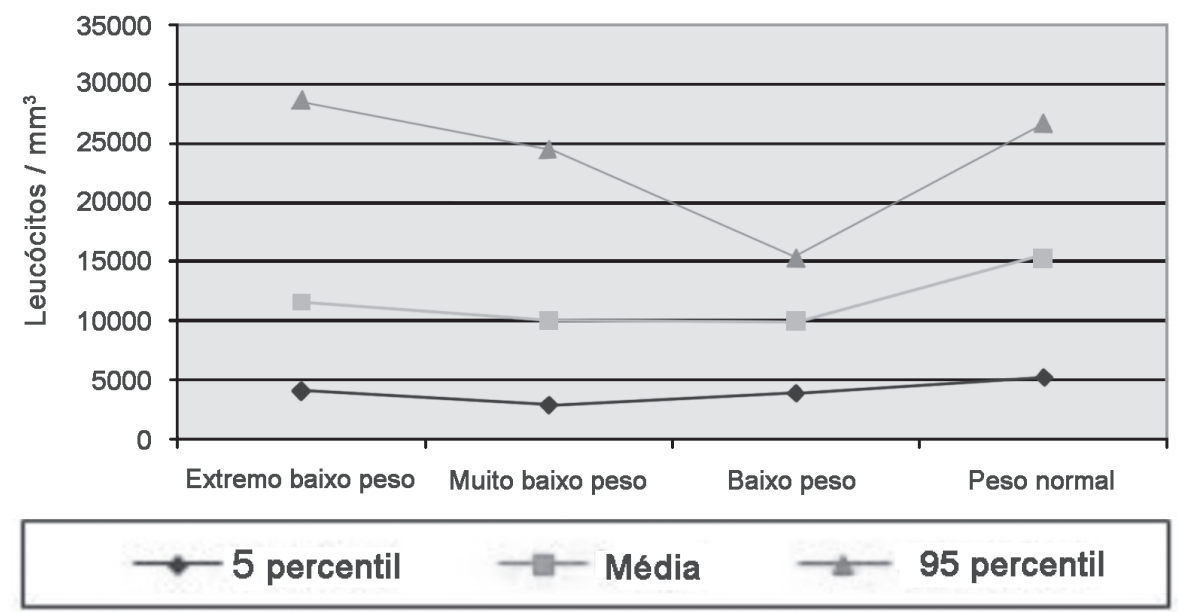

Figura 6. Faixas de referência estimadas para contagem de leucócitos totais $/ \mathrm{mm}^{3}$ por peso de nascimento $(p<0,05)$. Extremo baixo peso: $n=9$; muito baixo peso: $n=13$; baixo peso: $n=31$; peso normal: $n=44$

Tabela I. Faixas de referência estimadas para leucograma dos neonatos do HU/UFSC

\begin{tabular}{ccc}
\hline Células $/ \mathrm{mm}^{3}$ & $\begin{array}{c}\text { Média da } \\
\text { população estudada }\end{array}$ & $\begin{array}{c}\text { Faixa } \\
\text { estimada }\end{array}$ \\
\hline Leucócitos totais & 14.000 & $4.000-27.000$ \\
Mielócitos & 140 & $0-160$ \\
Metamielócitos & 250 & $0-600$ \\
Bastonados & 550 & $0-2.000$ \\
Neutrófilos & 7.300 & $1.800-19.000$ \\
segmentados & & \\
Linfócitos & 4.000 & $1.500-7.100$ \\
Monócitos & 1.200 & $250-2.900$ \\
Eosinófilos & 300 & $0-1.000$ \\
Basófilos & 40 & $0-160$ \\
\hline
\end{tabular}

uma média de 4 eritroblastos para cada 100 células na população, e a faixa de referência estimada foi de 0 a 18 eritroblastos para cada 100 leucócitos.

\section{Leucograma}

Para a análise dos glóbulos brancos, as faixas de referência foram estimadas baseadas na obtenção dos 5 e 95 percentuais, da mesma maneira que é estimada a maioria dos valores de referência para neonatos.1 Os leucócitos totais apresentaram diferença estatisticamente significativa entre as faixas de idade gestacional e peso de nascimento ( $\mathrm{p}<$ $0,05)$. Os valores encontrados estão representados pelas Figuras 5 e 6.

A média de leucócitos $/ \mathrm{mm}^{3}$ para os recém-nascidos de termo foi cerca de 2.000 leucócitos $/ \mathrm{mm}^{3}$ maior em comparação aos prematuros. Já para os neonatos de peso normal, essa média ficou cerca de 4.000 células $/ \mathrm{mm}^{3}$ acima da média estimada para os neonatos classificados nas demais faixas de peso.

$\mathrm{Na}$ análise de células imaturas também foi encontrada diferença estatisticamente significativa para as contagens de metamielócitos e bastonados entre recém-nascidos de diferentes idades gestacionais e peso de nascimento. As crianças com 28 semanas ou menos tiveram em média 160 metamielócitos/ $\mathrm{mm}^{3}$ a mais que aquelas com idade gestacional igual ou maior que 36 semanas. Em relação aos bastonados, a maior diferença foi obtida entre as crianças de extremo baixo peso e de peso normal, onde as primeiras apresentaram em média cerca de 270 células $/ \mathrm{mm}^{3}$ a menos em relação às outras.

Outra análise realizada foi a de neutrófilos totais, para a qual também foi encontrada diferença entre as faixas de idade gestacional e de peso. Em média, os neonatos com 28 ou menos semanas apresentaram cerca de 2.000 neutrófilos $/ \mathrm{mm}^{3}$ a menos que as crianças nascidas com 36 semanas ou mais. Para os recém-nascidos de extremo baixo peso, foi obtida uma diferença de quase 3.000 neutrófilos $/ \mathrm{mm}^{3}$ a menos em comparação aos de peso normal. Para os demais tipos celulares, não foram encontradas diferenças estatisticamente significativas entre as classificações.

Observando que as diferenças encontradas nos dados apresentados já estão descritas na literatura e são importantes para a interpretação do hemograma do recém-nascido, estes resultados não poderiam ser omitidos. Entretanto, consideramos prudente a apresentação de uma faixa de referência estimada para a população analisada como um todo, em relação ao leucograma completo, incluindo aqueles tipos celulares para os quais não ouve diferença significativa entre as populações. Os valores estão representados na Tabela I.

\section{Plaquetas}

Em nosso estudo, não houve diferença estatisticamente significativa para as contagens de plaquetas entre as faixas de idade gestacional e peso de nascimento. A faixa de referência obtida foi de 70.000 - 400.000 plaquetas $/ \mathrm{mm}^{3}$, sendo que a média da população analisada foi de 250.000 plaquetas $/ \mathrm{mm}^{3}$.

\section{Discussão}

A concentração de hemoglobina e o hematócrito estão entre os parâmetros mais solicitados pelos clínicos. ${ }^{3,9}$ Houve um aumento dos valores dos parâmetros eritrocitários estudados à medida que foi aumentando a idade gestacional dos neonatos. Aquelas crianças com 26 semanas ou menos tiveram a contagem de eritrócitos em média 1,5 milhão abaixo daquelas que nasceram de termo. No caso dos recém-nascidos de extremo baixo peso, as contagens foram em média 1 milhão 
abaixo quando comparadas aos neonatos com peso normal. Em 2005, Alarcón e colaboradores publicaram valores de referência de eritrócitos para recém-nascidos classificados de acordo com a idade gestacional. A faixa de referência para crianças entre 24 e 25 semanas foi de 4,65 $\pm 0,43$ hemácias $x$ $10-3 / \mathrm{mm}^{3}$ e de $5,14 \pm 0,7$ hemácias $x 10-3 / \mathrm{mm}^{3}$ para crianças de termo. ${ }^{8}$ Podemos observar o mesmo comportamento de ascensão dos parâmetros nos resultados obtidos pelo nosso estudo.

A dosagem de hemoglobina foi em média $5 \mathrm{~g} / \mathrm{dL}$ inferior e o hematócrito foi cerca de 10 pontos menor nos recémnascidos com 26 semanas ou menos quando comparados com os que nasceram com idade gestacional maior ou igual a 38 semanas. Em 2009, Christensen e colaboradores publicaram novas faixas de referência propostas para recém-nascidos, baseados em um estudo que usou grandes bases de dados com mais de 30 mil hemogramas de neonatos. Nesse trabalho, foi observado um valor de hematócrito até 10 pontos abaixo para os recém-nascidos com menos de 28 semanas quando comparados aos de termo. Também foi obtida uma faixa de referência para a hemoglobina $3,3 \mathrm{~g} / \mathrm{dL}$ abaixo para aquela população em relação às crianças com maior idade gestacional, resultados estes que corroboram com os obtidos pela nossa pesquisa.

Em 2001, Hermansen fez uma revisão sobre a contagem de eritroblastos no sangue periférico dos neonatos e, baseado em valores encontrados por vários pesquisadores, obteve uma faixa de referência de 0 a 10 eritroblastos para cada 100 leucócitos, pouco diferente da faixa encontrada pelo nosso estudo, que foi de 0 a 18 eritroblastos para cada 100 células. $\mathrm{O}$ autor conclui que o uso de um número absoluto de eritroblastos $/ \mathrm{mm}^{3}$ seria o ideal, pois o valor de hemácias nucleadas para cada 100 leucócitos é altamente dependente da variação das contagens de leucócitos totais. ${ }^{10}$

A estimativa de valores de referência de leucócitos totais para os recém-nascidos deve ser cautelosa, visto que os contadores automatizados incluem as hemácias nucleadas nesse número final. Um estudo com crianças nigerianas encontrou valores de leucócitos $/ \mathrm{mm}^{3}$ de $12.120 \pm 8.000$ para os recém-nascidos pré-termo e $12.230 \pm 5.490$ para os de termo. ${ }^{11}$ Em nosso trabalho foi feita a correção do número total de glóbulos brancos, e os valores encontrados foram ligeiramente diferentes dos anteriormente reportados.

Em 1979, Manroe e colaboradores apresentaram os primeiros valores de referência para neutrófilos em neonatos. ${ }^{12}$ Em 1994, Mouzinho e colaboradores publicaram um trabalho onde constataram que os valores anteriormente estabelecidos não se aplicavam aos recém-nascidos com peso de nascimento menor de $1.500 \mathrm{~g}$, e sugeriram que possivelmente as contagens de neutrófilos teriam influência do peso e idade gestacional da criança nas primeiras 60 horas de vida. ${ }^{5}$ Em 2008, Schmutz e seus colaboradores fizeram uma revisão desses valores, num estudo que usou 30.354 contagens diferenciais de neonatos. Nesse trabalho foram estabelecidas faixas de referência para recém-nascidos de acordo com sua idade gestacional. Aqueles com mais de 36 semanas se enquadraram entre 3.500-8.000 neutrófilos $/ \mathrm{mm}^{3}$; para neonatos com 28 a 36 semanas de idade gestacional, a faixa foi de 1.000 a 10.500 neutrófilos $/ \mathrm{mm}^{3}$; para aqueles com menos de 28 semanas, a faixa foi de 500 a 8.000 neutrófilos $/ \mathrm{mm}^{3} .{ }^{13} \mathrm{Em}$ nossa pesquisa, também observamos que as crianças com menor idade gestacional tiveram um limite inferior para a contagem de neutrófilos abaixo do limite inferior estimado para os neonatos de termo, indicando uma neutropenia comum a esses indivíduos, o que estaria de acordo com outros estudos já

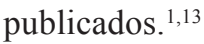

Em relação à contagem de plaquetas, diversos autores utilizam para os neonatos a mesma faixa de referência dos adultos, ${ }^{3,14}$ usualmente considerada entre 150.000-450.000 plaquetas $/ \mathrm{mm}^{3} .{ }^{14}$ Entretanto, publicações atuais apresentam diferentes faixas de referência para plaquetas nos recémnascidos. Em 2009, um estudo mostrou que as contagens de plaquetas podem frequentemente chegar até $650.000 / \mathrm{mm}^{3}$ dependendo da idade em dias do recém-nascido, o que poderia ser explicado por um pico fisiológico de trombopoetina detectado no sangue periférico dos neonatos por volta do segundo dia de vida. O mesmo estudo apresenta uma faixa de referência de cerca de 100.000 a 400.000 plaquetas por $\mathrm{mm}^{3}$ nas primeiras 72 horas de vida, ${ }^{1}$ em concordância com a faixa encontrada no nosso trabalho, que foi de 70.000 a 400.000 plaquetas $/ \mathrm{mm}^{3}$.

\section{Conclusão}

A interpretação do hemograma do recém-nascido deve ser feita com cautela, e dados como o peso de nascimento e a idade gestacional da criança devem ser considerados. Em nosso estudo obtivemos diferenças significativas de acordo com estes fatores para valores de eritrócitos, hemoglobina, hematócrito, leucócitos totais, neutrófilos imaturos e neutrófilos totais, diferenças essas que já haviam sido relatadas por outros pesquisadores. Estudos baseados em grandes populações de neonatos devem ser realizados para obtenção de faixas de referência confiáveis para os parâmetros que apresentam diferença entre as populações, auxiliando médicos e farmacêuticos-bioquímicos na realização e utilização dessa ferramenta diagnóstica tão importante que é o hemograma.

\footnotetext{
Abstract

The whole blood test of neonates provides much information with the interpretation changing according to the child's gestational age and birth weight. Reference values for this age can be hard to find as few studies have been made in this area in particular because of the difficulty in sampling and its implications for the newborn. This study compares the hematologic profile of neonates attended in a teaching hospital and compares the obtained values with reference
} 
ranges found in the literature. The results show significant differences compared to reference ranges found for many hematologic parameters, particularly related to the birth weight and gestational age of the newborns. This research demonstrates the importance of using different reference values for interpreting the whole blood test of neonates. Rev. Bras. Hematol. Hemoter. 2010;32(3):219-224.

Key words: Blood cell count; infant, newborn; reference values.

\section{Referências Bibliográficas}

1. Christensen RD, Henry E, Jopling J, Wiedmeier SE. The CBC: reference ranges for neonates. Semin Perinatol. 2009;33(1):3-11.

2. Marcondes E, Vaz FAC, Ramos JLA, Okay Y. Pediatria Básica. Tomo I. $9^{a}$ ed. São Paulo: Sarvier; 2003.

3. Failace R. Hemograma: Manual de interpretação. $4^{\mathrm{a}}$ ed. Porto Alegre: Artmed; 2003.

4. Ozyürek E, Cetintas S, Ceylan T, Ogüs E, Haberal A, Gürakan B, Ozbek N. Complete blood count parameters for healthy, smallfor-gestational-age, full-term newborns. Clin Lab Haematol. 2006;28(2):97-104.

5. Mouzinho A, Rosenfeld CR, Sánchez PJ, Risser R. Revised reference ranges for circulating neutrophils in very-low-birth-weight neonates. Pediatrics. 1994;94(1):73-82.

6. Horiba Group. ABX Pentra ML User manual CD-ROM, Software Version v.4.0x, 2007.

7. Borges LF, Siqueira LO. Validação de tecnologia 5 diff do analisador hematológico Sysmex XS-1000i para laboratório de pequeno/médio porte. Rev. Bras. Hematol. Hemoter. 2009;31(4):247-51.

8. Alarcón PA, Werner EJ. Normal Values and laboratory methods In: Neonatal Hematology. New York: Cambrigde University Press, 2005.

9. Buttarello M, Plebani M. Automated blood cell counts: state of the art. Am J Clin Pathol. 2008;130(1):104-16.

10. Hermansen MC. Nucleated red blood cells in the fetus and newborn. Arch Dis Chils Fetal Neonatal Ed. 2001;84(3):F211-15.

11. Scott-Emuakpor AB, Okolo AA, Omene JA, Ukpe SI. Pattern of leukocytes in the blood of healthy African neonates. Acta Haematol. 1985;74(2):104-7.

12. Manroe BL, Weinberg AG, Rosenfeld CR, Browne R. The neonatal blood count in health and disease. I. Reference values for neutrophilic cell. J Pediatr 1979;95(1):89-98.

13. Schmutz N, Henry E, Jopling J, Christensen RD. Expected ranges for blood neutrophil concentration of neonates: the Manroe and Mouzinho charts revisited. J Perinatol. 2008;28(4):275-81.

14. Lewis SM, Bain BJ, Bates I. Hematologia prática de Dacie e Lewis. $9^{\text {a }}$ Ed. Porto Alegre: Artmed, 2003.

Avaliação: Editor e dois revisores externos

Conflito de interesse: sem conflito de interesse

Recebido: 10/12/2009

Aceito após modificações: 27/05/2010 